\title{
On the Biological Plausibility of Artificial Metaplasticity ${ }^{\star}$
}

\author{
Diego Andina and Javier Ropero-Peláez \\ Group for Automation in Signal and Communications, Technical University of \\ Madrid, Spain and \\ Federal University of A.B.C, Brazil
}

\begin{abstract}
The training algorithm studied in this paper is inspired by the biological metaplasticity property of neurons. Tested on different multidisciplinary applications, it achieves a more efficient training and improves Artificial Neural Network Performance. The algorithm has been recently proposed for Artificial Neural Networks in general, although for the purpose of discussing its biological plausibility, a Multilayer Perceptron has been used. During the training phase, the artificial metaplasticity multilayer perceptron could be considered a new probabilistic version of the presynaptic rule, as during the training phase the algorithm assigns higher values for updating the weights in the less probable activations than in the ones with higher probability.
\end{abstract}

\section{Introduction}

Artificial Metaplasticity (AMP) term was first introduced by Andina et al [1] for an Artificial Neural Network (ANN) of the Multilayer Perceptron type (MLP), referred as AMMLP. During the AMMLP training phase, the matrix weight $W$ that models the synaptic strength of its artificial neurons is updated according to the probability of the input patterns and therefore of the corresponding synaptic activations. The concept of biological metaplasticity was defined in 1996 by W.C. Abraham [2] and now is widely applied in the fields of biology, neuroscience, physiology, neurology and others $[2,3,4]$. The prefix "meta" comes from Greek and means "beyond" or "above". In neuroscience and other fields "metaplasticity" indicates a higher level of plasticity, expressed as a change or transformation in the way synaptic efficacy is modified. Metaplasticity is defined as the induction of synaptic changes, that depends on prior synaptic activity [3,5]. Metaplasticity is due, at least in part, to variations in the level of postsynaptic depolarization that induce synaptic changes. These variations facilitate synaptic potentiation and inhibit synaptic depression in depressed synapses (and vice versa in potentiated synapses). The direction and the degree of the synaptic alteration are functions of postsynaptic depolarization during synaptic activation. Upregulation -incrementing, reinforcement of synaptic efficacy- is termed

\footnotetext{
* This research has been supported by Group for Automation in Signal and Communications, GASC/UPM.
} 
long-term potentiation (LTP), whereas downregulation -decrementing inhibingis known as long-term depression (LTD). LTP and LTD are believed to be fundamental to storage of memory in the brain and hence learning.

The induction of synaptic changes in the levels of neural activity is explained [6] in Fig. 1. Metaplasticity can be represented as variations in curve elongation with respect to the level of activity and implies a shift of the LTP threshold according to the weight strength of the synapse [6]. Fig. 1 graphically illustrate this idea. Understanding metaplasticity may yield new insights into how the modification of synapses is regulated and how information is stored by synapses in the brain. $[7,8,9]$

This paper is organized as follows. Section 2 provides a brief introduction to related concepts (e.g. synaptic plasticity). Main postulation regarding the relation between metaplasticity and Shannon's Information Theory is introduced in Section 3, to ease the understanding of the proposed model. In Section 4, general mathematical theory is applied to describe the proposed implementation of AMP in a MLP, whose learning process is based on error minimization. In Section 5, we implement the AMP algorithm in the MLP neural network, which is trained with the $\mathrm{BP}$ algorithm with a modified objective function. Section 6 presents a discussion on the biological plausibility of the AMMLP algorithm, refering to experimental results. Finally, Section 7 summarizes main conclusions.

\section{Synaptic Plasticity and Metaplasticity}

Synaptic plasticity refers to the efficacy modulation of information transmission between neurons, being related to the regulation of the number of ionic channels in synapses. Synaptic plasticity mechanisms involve both molecular and structural modifications that affect synaptic functioning, either enhancing or depressing neuronal transmission. They include redistribution of postsynaptic receptors, activation of intracellular signaling cascades, and formation/retraction of the dendrites [10]. The first model of synaptic plasticity was postulated by Hebb and it is known as the Hebb rule [11].

In Fig.1, the effect of metaplasticity is illustrated. This graphic shows a family of curves in which each curve indicates the biological variation in weight, $\Delta \omega$, respective of the neurons activation frequency or postsynaptic activity. If postsynaptic activity is high, by metaplasticity property, the curve will move to the right, reinforcing the LTP. Andina postulates [1] that high postsynaptic activity corresponds to high frequent excitations -frequent input classes in an artificial model-. In the same way, the left-hand side curves of the family corresponding to low previous synaptic activity correspond to low frequent excitations produced by patterns of unfrequent classes. During training, postsynaptic activity is the same for each training pattern -one excitation by epoch-. As it can be observed in Fig.1, for a given postsynaptic activity value, a higher $\Delta \omega$ corresponds to the un-frequent classes curves than to the curves corresponding to frequent ones. 




Fig. 1. Heterosynaptic BCM model [21] illustrates changes in synaptic strength due to postsynaptic activity in biological neurons

\subsection{Intrinsic Plasticity}

A third concept about biological plasticity is the intrinsic plasticity, and it must not be misunderstood with the previous ones. When neurons are receiving and responding to synaptic inputs, the synaptic metaplasticity makes it difficult for synaptic weights to become either null or saturated. But the metaplasticity property cannot fully avoid these two extreme situations. For totally precluding the possibility of either weight annihilation or saturation, another important homeostatic property of real neurons should be taken into account: the so-called intrinsic plasticity [12]. Intrinsic plasticity regulates the position (rightward shift) of neurons activation function, according to previous levels of activity [6]. Intrinsic plasticity is not modelled in the AMMLP.

\section{Metaplasticity and Shannon Information Theory}

As is well-known within the ANN field, in 1949 Hebb postulated that during the learning phase, synaptic connections between biological neurons are strengthened due to the correlated activity of presynaptic and postsynaptic neurons [11]. This plasticity property of synaptic connections is modeled in many ANNs as a change in the connection weights of the artificial neurons or nodes. Therefore, synaptic plasticity of biological neural networks has been simulated in artificial networks by changing the weight values of the simulated neuronal connections. These weights are the most relevant parameters in ANN learning and performance. Modeling these new discovered properties of biological neurons that follow metaplasticity rules provides a large potential for improving ANN learning. In addition, the results of these simulations may also corroborate the biological hypothesis of neuronal metaplasticity. Utilizing the potential of this new modeling approach, artificial metaplasticity (AMP) models have been devised and tested. A model that closely followed biological metaplasticity and intrinsic plasticity was successfully tested in the reinforcing the calcium dysregulation hypothesis for Alzheimer's disease [13]. However, of all AMP models tested by 
the authors, the most efficient model (as a function of learning time and performance) is the approach that connects metaplasticity and Shannon information theory, which establishes that less frequent patterns carry more information than frequent patterns [14]. This model then defines artificial metaplasticity as a learning procedure that produces greater modifications in the synaptic weights with less frequent patterns than frequent patterns, as a way of extracting more information from the former than from the latter. As Biological metaplasticity, AMP then favors synaptic strengthening for low-level synaptic activity, while the opposite occurs for high level activity. The model is applicable to general ANNs. Andina et al. propose general AMP concepts for ANNs, and demonstrate them over Radar detection data [1].

\section{Backpropagation Algorithm and AMP}

The AMP implementation applied tries to improve results in learning convergence and performance by capturing information associated with significant rare events. It is based on the idea of modifying the ANN learning procedure such that un-frequent patterns which can contribute heavily to the performance, are considered with greater relevance during learning without changing the convergence of the error minimization algorithm. It is has been proposed on the hypothesis that biological metaplasticity property maybe significantly due to an adaptation of nature to extract more information from un-frequent patterns (low synaptic activity) that, according to Shannon's Theorem, implicitly carry more information.

\subsection{Mathematical Definitions}

Let us define an input vector for a MLP with $n$ inputs (bias inputs are assumed to exist and be of fixed value set to 1$): x \in R^{n}$, where $R^{n}$ is the n-dimensional space, i.e. $x=\left(x_{1}, x_{2}, \ldots, x_{n}\right), x_{i} \in R^{1}, i=1,2, \ldots, n$; and its corresponding $j$ outputs given by vector $y=\left(y_{1}, y_{2}, \ldots, y_{n}\right), y_{i} \in(0,1), j=1,2, \ldots, m$ [15]. Let us consider now the random variable of input vectors $X=\left(X_{1}, X_{2}, \ldots, X_{n}\right)$ with probability density function ( $p d f) f_{X}(x)=f_{X}\left(x_{1}, x_{1}, \ldots, x_{n}\right)$. The strategy of MLP learning is to minimize an expected error, $E_{M}$, defined by the following expression:

$$
E_{M}=\varepsilon\{E(x)\}
$$

where $E(x)$ is the expression of an error function between the real and the desired network output, being respectively $Y=F(X)$, with pdf $f_{Y}(y)$ and $Y_{d}$ the desired output vector, and $F(X)$ is the nonlinear function performed by the MLP. The symbol $\varepsilon$ represents the mathematical expectation value, that is,

$$
E_{M}=\int_{R^{n}} E(x) f_{X}(x) d x
$$




\subsection{AMP in Gradient Descent Algorithm}

BackPropagation training algorithm applied in MLPs follows Widrow gradient descent algorithm over an estimation of this expected error in each training iteration, $t \in N$, for determining the necessary modification in the ANN weight matrix $W(t)$ in each bias and weight value in the MLP [15]. The algorithm objective is to reduce the output classification error in subsequent training epochs, stopping the training phase if the error is low enough to satisfy the design requirements.

To introduce AMP in the gradient descent algorithm, let us define

$$
e(x) \equiv E(x) f_{X}(x)
$$

and manipulate Eq. (2) in the following way:

$$
\begin{aligned}
E_{M}= & \int_{R^{n}} \frac{e(x) f_{X}^{*}(x)}{f_{X}^{*}(x)} d x= \\
& =\varepsilon^{*}\left\{\frac{e(x)}{f_{X}^{*}(x)}\right\}
\end{aligned}
$$

where a new probability density function (pdf) $f_{X}^{*}(x)$ has been introduced, requiring that $f_{X}^{*}(x) \neq 0$ wherever $e(x) \neq 0, \forall x \in R^{n}$ and new mathematical expectation, $\varepsilon^{*}$, defined in Eq. (3) represents that the minimization of $E_{M}$ can also be achieve from statistical inference theory applied to Eq. (3), by estimating over the weighted function $e(x) / f_{X}^{*}(x)$ instead of $e(x)$, under $f_{X}^{*}(x)$ pdf, through the estimator $\hat{E}_{M}$, defined as:

$$
\hat{E}_{M} \equiv \frac{1}{P} \sum_{k=1}^{P} \frac{e\left(x_{k}^{*}\right)}{f_{X}^{*}\left(x_{k}^{*}\right)}
$$

where $x_{k}^{*}, k=1,2, \ldots, P$, are independent sample vectors whose pdf is the weighting function $f_{X}^{*}(x)$. Note that many functions may fix to the definition of $f_{X}^{*}(x)$, in particular:

$$
\left[f_{X}^{*}(x)\right]_{o p t}=\frac{1}{E_{M}} e(x)
$$

that can be proved by taking Eq. (5) into Eq. (4); only one simple sample vector $(P=1)$ is then required for exactly estimating $E_{M}$ without error. The optimal solution for $f_{X}^{*}(x)$ given by Eq. (5) is not realizable, because $E_{M}$ is not known a priori (it has to be estimated by Eq. (4)). But, a suboptimal solution can be used. For example, a common suboptimal solution for $f_{X}^{*}(x)$ applied and tested succesfully in many applications is:

$$
f_{X}^{*}(x)=\frac{A}{\sqrt{(2 \pi)^{N}} \cdot e^{B \sum_{i=1}^{N} x_{i}^{2}}} \equiv \frac{1}{w_{X}^{*}(x)}
$$


where $w_{X}^{*}(x)$ is defined as $1 / f_{X}^{*}(x), N$ is the number of neurons in the MLP input layer, and parameters $A$ and $B \in R^{+}$are algorithm optimization values which depend on the specific application of the AMLP algorithm. Values for $A$ and $B$ have been empirically determined. Eq. (6) is a gaussian distribution, so it has been assumed that $X$ pdf is Gaussian (if it is not the case, the real $X$ pdf should be used instead). Then, $w_{X}^{*}(x)$ has high values for un-frequent $\mathrm{x}$ values and close to 1 for the frequent ones and can therefore be straightforwardly applied in weights updating procedure to model the biological metaplasticity during learning.

\section{AMP in MLP Training: AMMLP}

In the case of an MLP trained with BPA applied to $L$ classes, $H_{l}, l=0,1, \ldots, L-1$, previous studies have shown that the output for each class is the MLP inherent estimation of a posteriori probability of the class [16], based on Bayes Theorem, we then have:

$$
y_{l} \cong P\left(H_{l} \mid x\right)=\frac{f_{X}\left(x \mid H_{l}\right) \cdot P\left(H_{l}\right)}{f_{X}(x)}
$$

This enables a direct implementation of metaplasticity. For each class, by assuming the proposed AMP model described in subsection 4.2 can be make $f_{X}^{*}(x)=f_{X}(x)$ and from Eq. (7) and Eq. (4)

$$
\begin{gathered}
e\left(x \mid H_{l}\right) E(x) f_{X}\left(x \mid H_{l}\right) \\
\hat{E}_{M_{l}}=\frac{1}{M_{l}} \sum_{k=1}^{M_{l}} \frac{E\left(x_{k}\right) f_{X}\left(x \mid H_{l}\right)}{f_{X}\left(x_{k}\right)} \\
\cong \frac{1}{M_{l}} \sum_{k=1}^{M_{l}} E\left(x_{k}\right) \frac{y_{l}}{P\left(H_{l}\right)}
\end{gathered}
$$

where $k=1,2 \ldots, M_{l}$, are the independent sample vectors of class $l$ in the training set. Then, from Eq. (8) and Eq. (4)

$$
\frac{y_{l}}{P\left(H_{l}\right)} \cong \frac{1}{f^{*}(x)}
$$

Eq. (7) takes advantage of the inherent a posteriori probability estimation for each input class of MLP outputs, so it is used to quantify a pattern's frequency. Note that if this is not the case, as it happens in first steps of BPA training algorithm, the training may not converge. In this first steps, the outputs of the MLP does not provide yet any valid estimation of the a posteriori probabilities, but rather random values corresponding to initial guess of the MLP weights, $W$ . It is then better in these first steps of training, either to apply ordinary BPA training or to use another valid weighting function till BPA starts to minimize 
the error objective. Also, many suboptimal functions may yield good results. For example, in the following experiments, a typical approximation premise that assumes a Gaussian distribution for the inputs has been implemented, proposing the function for weight updating (known as a weighting function) [1], given by Eq. (6).

To analytically introduce AMP in an arbitrary MLP training, all that has to be done is to introduce the weighting function in the error function between the real and the desired network output, as a function of the weights matrix $W(t)$ in each training iteration, $t$, that is

$$
E^{*}[W(t)]=\frac{E[W(t)]}{f_{X}^{*}(x)}
$$

And apply the BPA [15] to the weighted error $E^{*}(W)$ for weights reinforcement in each iteration $t \in \mathrm{N}$. If $s, j, i \in N$ are the MLP layer, node and input counters respectively, for each $W(t)$ component, $w_{i j}^{(s)}(t) \in R$, and being $\eta \in R^{+}$the learning rate, then the weight reinforcement in each iteration is given by:

$$
\begin{array}{r}
w_{i j}^{(s)}(t+1)=w_{i j}^{(s)}(t)-\eta \frac{\partial E^{*}[W(t)]}{\partial w_{i j}^{(s)}} \\
=w_{i j}^{(s)}(t)-\eta \frac{1}{f_{X}^{*}} \frac{\partial E[W(t)]}{\partial w_{i j}^{(s)}}
\end{array}
$$

So, as the pdf weighting function proposed is the distribution of the input patterns that does not depend on the network parameters, the AMMLP algorithm can then be summarized as a weighting operation for updating each weight in each MLP learning iteration as:

$$
\Delta^{*} w=w^{*}(x) \Delta w
$$

being $\Delta w=w(t+l)-w(t)$ the weight updating value obtained by usual BPA and $w^{*}(x)$ the realization of the described weighting function $w^{*}(x)$ for each input training pattern $x$. During the training phase, the artificial metaplasticity multilayer perceptron could be considered a new probabilistic version of the presynaptic rule, as during the training phase the algorithm assigns higher values for updating the weights in the less probable activations than in the ones with higher probability.

\section{Discussion}

Plasticity and Metaplasticy still pose a considerable challenge for research in terms of experimental design and interpretation [22]. Along the years, different mathematical models of synaptic computation have been proposed. In the classical Hebb model [11], the curve relating the increment of synaptic weight to postsynaptic activation is a straight line without synaptic depression. In Sejnowski's covariance model $[23,24]$, regions of potentiation and depression are 
separated by a LTP threshold. In [25] Abraham and Bear consider it as a homosynaptic property (i.e. involving only the synapse under study, without the need of considering the influence of nearby synapses). Whereas the model by Bienenstock, Cooper and Munro [21] yields a curve that is closer to reality, with LTP threshold determined by postsynaptic activation and without LTD threshold. In the BCM model, the LTP threshold is the same for all neuron synapses, so that metaplasticity would affect even non-active synapses (heterosynaptic plasticity). But despite its unquestionable biological characteristics, the BCM model, cannot be regarded to be the ultimate model of synaptic metaplasticity. According to Mockett and colleagues [27] metaplasticity is inherently a homosynaptic phenomenon in contrast to the heterosynaptic nature of the BCM rule. Finally, Artola, Bröcher and Singer's [28]) extended model (ABS model) is not analytical, as those just discussed, but is based on empirical experimental data. In the ABS model, LTP and LTD thresholds shift to lower values for higher levels of the activation of neighbouring synapses.

In its implementation characteristics, the proposed AMP model follows closer to the BCM model. We do not pretend to determine its superiority, but neurobiology inspires computer science and vice versa, and we report that the empirical results of AMP show a great potential, in terms of improving learning and therefore performance in most cases, no matter in what multidisciplinary application is applied $[1,17,18,28]$.

\section{Conclusion}

We describe and discuss the biological plausibility of an artificial model of metaplasticity, a relevant property of neurons. Tested on different multidisciplinary applications, it achieves a more efficient training and improves Artificial Neural Network Performance. The model follows the BCM heterosynaptic biological model. During the training phase, the artificial Artificial Metaplasticity Multilayer Perceptron could be considered a new probabilistic version of the presynaptic rule, as it assigns higher values for updating the weights in the less probable activations than in the ones with higher probability.

\section{References}

1. Andina, D., Alvarez-Vellisco, A., Jevtic, A., Fombellida, J.: Artificial metaplasticity can improve artificial neural network learning. In: Intelligent Automation and Soft Computing, SI on Signal Processing and Soft Computing, vol. 15, pp. 683-696 (2009), ISSN: 1079-8587

2. Abraham, W.C.: Activity-dependent regulation of synaptic plasticity (metaplasticity) in the hippocampus. In: Kato, N. (ed.) The Hippocampus: Functions and Clinical Relevance, pp. 15-26. Elsevier, Amsterdam (1996)

3. Abraham, W.C., Bear, M.F.: Metaplasticity: The plasticity of synaptic plasticity. Trends in Neurosciences 19, 126-130 (1996), doi:10.1016/S0166-2236(96)80018-X 
4. Kinto, E., Del-Moral-Hernandez, E., Marcano-Cedeño, A., Ropero-Pelaez, J.: A preliminary neural model for movement direction recognition based on biologically plausible plasticity rules. In: Proc. 2nd Int. Work-Conf. on the Interplay between Natural and Artificial Computation, Lecture Notes in Computer Science, pp. 628-636. Springer, Berlin (2007)

5. Chiappalone, M., Vato, A., Berdondini, L., Koudelka, M., Martinoia, S.: Network Dynamics and Synchronous Activity in Cultured Cortical Neurons. Int J. Neural Syst. 17(2), 87-103 (2007)

6. Ropero-Pelaez, J., Piqueira, J.R.: Biological clues for up-to-date artificial neurons. In: Computational Intelligence for Engineering and Manufacturing, pp. 131-146. Springer, Heidelberg (2007)

7. Bolle, D., Heylen, R.: Adaptive Thresholds for Neural Networks with Synaptic Noise. Int J. Neural Syst. 17(4), 241-252 (2007)

8. Abraham, W.C.: Metaplasticity: tuning synapses and networks for plasticity. Nature Reviews Neuroscience 9, 387-399 (2008), doi:10.1038/nrn2356.

9. Daoudal, G., Debanne, D.: Long-Term Plasticity of Intrinsic Excitability: Learning Rules and Mechanisms. Learning \& Memory. Nature Reviews Neuroscience 10, 456-465 (2003), doi:10.1101/lm.65303

10. Neves, G., Cooke, S.F., Bliss, T.V.: Synaptic plasticity, memory and the hippocampus: a neural network approach to causality. Nature Rev. Neurosci. 9, 65-75 (2008), doi:10.1038/nrn2303.

11. Hebb, D.O.: The Organization of Behavior. In: Mahwah, N.J. (ed.) Reedition of the 1949 original (2002) ISBN-10: 0805843000, ISBN-13: 978-0805843002

12. Cudmore, R.H., Turrigiano, G.G.: Long-Term Potentiation of Intrinsic Excitability in LV Visual Cortical Neurons. Journal Neurophysiology 92, 341-348 (2004), doi:10.1152/jn.01059.2003.

13. Monteiro, J.L., Lobo-Netto, M., Andina, D., Pelaez, J.R.: Using Neural Networks to Simulate the Alzheimer's Disease. In: Davis, C.G., Yeh, R.T. (eds.) Proc. World Automation Congress, Hawaii, HI, USA, pp. 1-6 (2008) ISBN: 978-1-889335-38-4. INSPEC Accession Number: 10411864

14. Shannon, C.E.: A mathematical theory of communication. The Bell System Technical Journal 27, 379-423 (1948)

15. Andina, D., Pham, D.T. (eds.): Computational Intelligence for Engineering and Manufacturing. Springer, Heidelberg (2007)

16. Rucky, D.W., Rogers, S.K., Kabrisk, M., Oxley, M.E., Suter, B.W.: The multi-layer perceptron as an approximation to a Bayes optimal discrimination function. IEEE Transactions on Neural Networks 1, 296-298 (1990), doi:10.1109/72.80266.

17. Marcano-Cedeño, A., Âlvarez-Vellisco, A., Andina, D.: Artificial metaplasticity MLP applied to image classification. In: Proc. 7th Int. Conf. on Industrial Informatics, Cardiff, United Kingdom, pp. 650-653 (2009), doi:10.1109/INDIN.2009.5195879.

18. Marcano-Cedeño, A., Quintanilla-DomÍnguez, J., Andina, D.: Wood Defects Classification Using Artificial Metaplasticity Neural Network. In: Proc. 35th Annua Conf. on of the IEEE Industrial Electronics Society, Porto, Portugal, pp. 3422-3427 (2009), doi:10.1109/IECON.2009.5415189

19. De Long, E., Clarke-Pearson, D.: Comparing the areas under two or more correlated receiver operating characteristic curves: A nonparametric approach. Biometrics 44, $837-845$ (1988)

20. Egan, J.: Signal Detection Theory and ROC analysis. In: Series in Cognition and Perception, SAcademic Press, New York (1975) 
21. Bienestock, E.L., Cooper, L.N., Munro, P.W.: Theory of the developement of neuron selectivity: orientation specifity and binocular interaction in visual cortex. J. Neurosci. 2(1), 32-48 (1982)

22. Abraham, W.C.: Metaplasticity: tuning synapses and networks for plasticity. In: Nature Reviews/neuroscience, vol. 9, pp. 387-399. Nature Publishing Group ( May 2008)

23. Sejnowski, T.J.: Storing covariance with non linearly interacting neurons. J. Math. Biol. 4, 303-321 (1977)

24. Sejnowski, T.J., Chattarji, S., Stanton, P.K.: Homosynaptic long-term depression in hippocampus and neocortex. Seminars in the Neurosciences 2, 355-363 (1990)

25. Abraham, W.C., Bear, W.C.: Metaplasticity the plasticity of synaptic plasticity. Trends Neurosci. 19, 126-130 (1996)

26. Mockett, B., Coussens, C., Abraham, W.C.: NMDA receptor-mediated metaplasticity during the induction of long-term depression by low-frequency stimulation. European Journal of Neuroscience 15, 1819-1826 (2002)

27. Artola, A., Singer, W.: Long-term depression of excitatory synaptic transmission and its relationship to long-term potentiation. Trends Neurosci. 16, 480-487 (1993)

28. Marcano-Cedeño, A., Quintanilla-Domínguez, J., Andina, D.: Breast Cancer Classification applying Artificial Metaplasticity Algorithm. In: Neurocomputing, Corrected Proof, pp. 925-2312. Elsevier, Amsterdam (2010), (in press) doi:10.1016/j.neucom.2010.07.019 